\title{
Bacterial etiology of chronic osteomyelitis involving anaerobes
}

\author{
Haitham M Al-Habib*, Mahmood A Aljumaily** \\ *Department of Microbiology, **Department of Surgery, College of Medicine, University of Mosul.
}

(Ann. Coll. Med. Mosul 2010; 36 (1 \& 2): 106-113).

Received: $21^{\text {st }}$ Jul 2010; Accepted: $12^{\text {th }}$ Jan 2011.

\begin{abstract}
Objective: To identify common bacterial causes in chronic osteomyelitis, to verify the role of anaerobic microorganisms in chronic osteomyelitis, and to assess the reliability of swab from discharging sinuses, and from abscess aspirate in chronic osteomyelitis.

Material and Methods: The study included 184 patients suffering from chronic osteomyelitis, 141 males and 43 females, ranging in age between 4-78 years with mean 40.5 years. The patients presented with chronic discharging sinus in 152 patients or acute flare up in 32 patients; they were divided according to causes into two broad categories haematogenous in 52 patients and exogenous in 132 patients. Specimens were taken from infected bone debridement during surgery, other specimens taken from sinus discharge or aspirated from bone abscesses. All specimens were inoculated on different culture media and incubated both aerobically and anaerobically.

Results: The majority of cases of chronic osteomyelitis involved long bones specially the femur and tibia (64.6\%). Aerobic microorganisms were found in 134 patients (73\%), staph. aureus and pseudomonas were the commonest aerobic isolates. In fifty cases (27\%), the causative microorganisms were anaerobic. Anaerobes were found to be mixed with aerobes in 34 cases $(68 \%)$, while pure anaerobes were obtained in 16 cases (32\%). Peptostreptococcus and Bacteroides were the dominant anaerobic isolates. The reliability of culture of abscesses aspirate in comparison with bone debridement culture is $93.3 \%$. The reliability of culture of sinus discharge in comparison with bone debridement culture is $42.7 \%$, while for anaerobic infection is $26 \%$.

Conclusion: Chronic osteomyelitis commonly affect adult males in long bones. The exogenous causes were the commonest. Aerobic isolates were the major cause, and anaerobic bacteria were an important other causative agents. Anaerobes usually isolated in prolonged persistent infection and pure anaerobes in older patients. The cultures of aspirate from bone abscesses were reliable way in diagnosis, while cultures from discharging sinuses had low diagnostic reliability and lower for anaerobic infection.
\end{abstract}

Keywords: Osteomyelitis, chronic, aerobic, anaerobic, microorganisms.

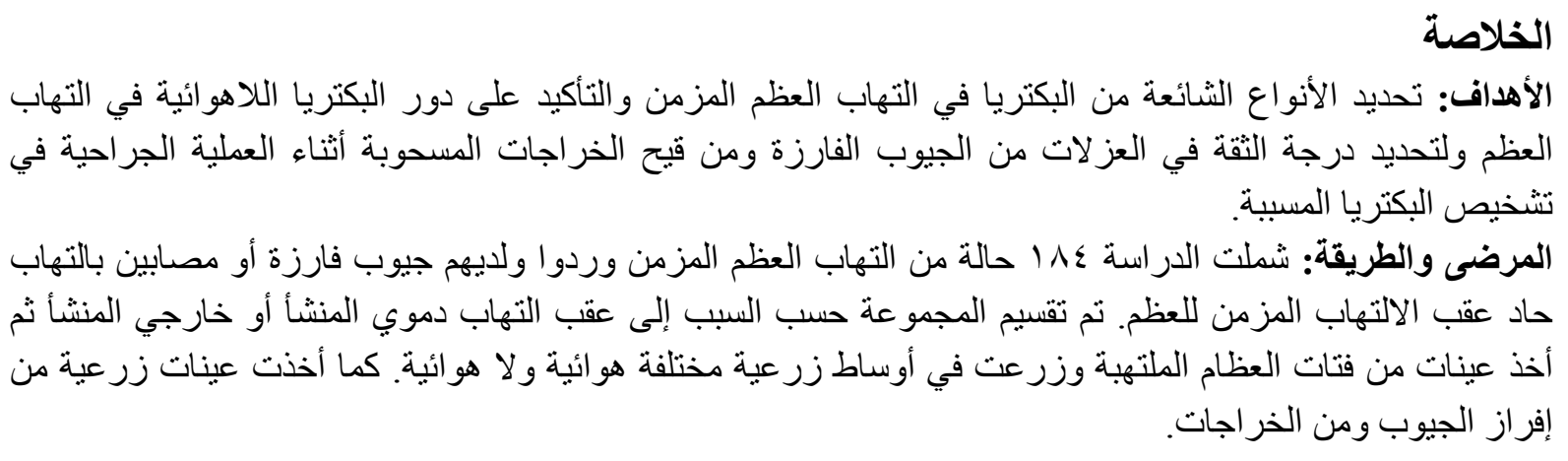




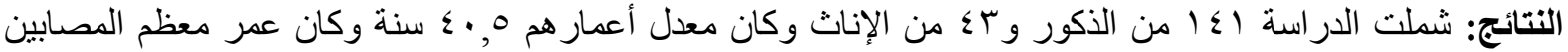

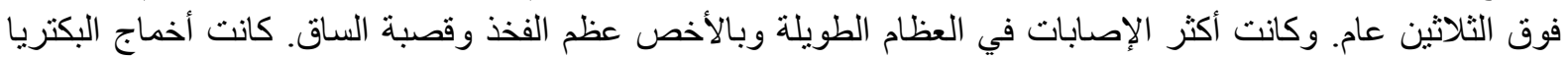

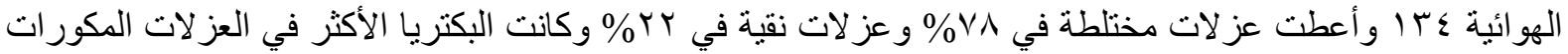

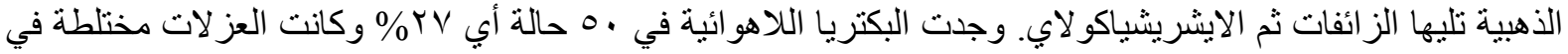

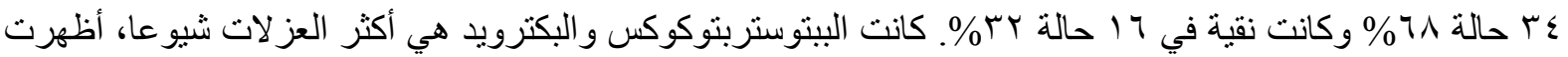

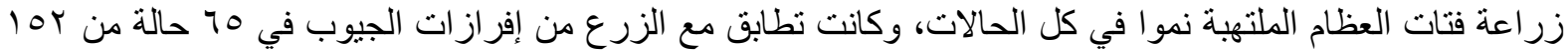

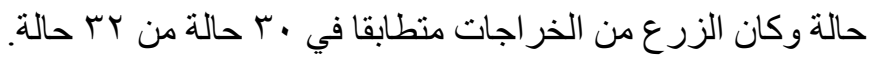

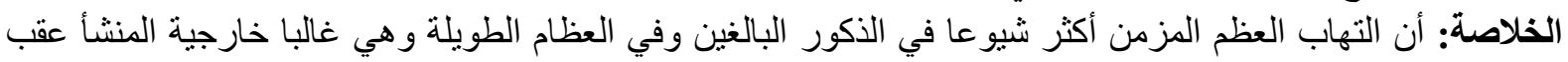

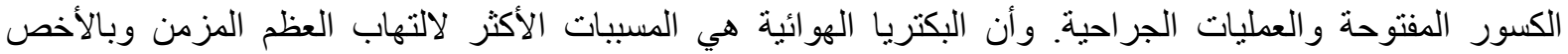

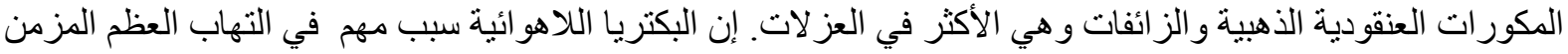

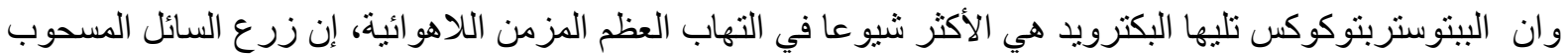

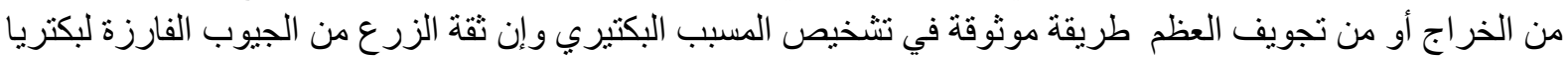
قليلة واقل في حالة البكتريا اللاهو ائية.

$\mathrm{O}$ steomyelitis is an infection of bone and bone marrow that may be caused by direct inoculation of open fractures or surgical procedures (exogenous), or blood born organism (haematogenous) ${ }^{(1)}$. Osteomyelitis could be acute or chronic although distinction between the two types may not be clear either clinically or on morphologic examination of tissue ${ }^{(2,3)}$. Chronic osteomyelitis used to be the dreaded sequel of acute haematogenous osteomyelitis; nowadays it more frequently follows open fractures or postoperative ${ }^{(4)}$. Chronic osteomyelitis is difficult to eradicate completely, systemic symptoms may subside, but one or more foci in the bone may contain purulent material, infected granulation tissues or a squestrum ${ }^{(5)}$. Intermittent acute exacerbations may occur for years ${ }^{(5)}$.

Culture of material taken from a bone biopsy is needed to identify the specific pathogens, alternative sampling methods such as needle puncture or surface swabs are easier to perform ${ }^{(6)}$. the usual aerobic organisms in chronic osteomyelitis are Staph. aureus, Escherichia coli, Streptococcus pyogenes, Proteus, and Pseudomonas ${ }^{(4)}$.

Anaerobic bacteria are recognized increasingly as an important cause of osteomyelitis. The first case of anaerobic osteomyelitis was reported by Vone Lanyebeck $^{(7)}$. Purely anaerobic osteomyelitis resulting from haematogenous spread is rare
$(1,2,3)$. The most frequent anaerobic isolates from cultures are Bacteroides species or the gram positive anaerobic cocci Peptostreptococcus ${ }^{(8)}$. Osteomyelitis caused solely by a clostridial species in pure culture is uncommon ${ }^{(9)}$. Anaerobic osteomyelitis of the feet is usually associated with diabetes mellitus, severe trauma or other underlying diseases causing vascular insufficiency ${ }^{(10)}$.

The aim of this study was to identify bacterial isolates from infected bone debridement cultures of chronic osteomyelitis including the anaerobes, and to assess the reliability of cultures taken from discharging sinuses, and those obtained by aspiration from subperiosteal and medullary abscesses.

\section{Patients and methods}

This study was approved by the scientific research committee at the College of Medicine, University of Mosul. Formal consent was taken from all patients after careful explanation. The current study was conducted at Al-Jumhori Teaching Hospital in Mosul and the department of microbiology, College of Medicine, University of Mosul, during the period from October 2003 to April 2010. One hundred and eighty four (184) cases of chronic pyogenic osteomyelitis were investigated for causative microorganisms including anaerobic bacteria by culturing infected bone debridement taken during surgery. There were 141 males and 43 females; males to females 
ratio was 3.3:1, their mean age was 40.5; (range 6-78 years). The age and sex distribution of the patients are presented in (table 1). The cases were divided clinically according to the causes into chronic osteomyelitis following acute haematogenous osteomyelitis (haematogenous) in 52 patients (28.3\%), and exogenous (posttraumatic and postoperative) in 132 patients (71.7\%). In 152 patients, the presentation was chronic discharging sinuses, samples of pus for cultures were taken before surgery from sinuses, in addition to the samples obtained from infected bone debridement during surgery. In 32 cases, the presentation was acute on chronic osteomyelitis, sample of pus aspirated during surgery by sterile disposable syringe from subperiosteal abscesses or medullary abscesses, in addition to the samples taken from infected bone debridement during surgery. Antibiotic stopped at least for three days before surgery and sample collection.

\section{Microbiological methods}

Each specimen was inoculated directly on to chocolate agar plate and blood agar base plate containing $5 \%$ sheep blood that had been held in anaerobic jar with gas pak generating kit (Oxoid, U.K.). Another blood agar plate (incubated aerobically in 5\% CO2 atmosphere) and MacConkey's agar were used for isolation of other aerobic microorganism. In addition other culture media were employed for the isolation of anaerobic bacteria, namely sodium thioglycolate broth, cooked meat medium, and brain heart infusion. The inoculation of the appropriate culture media was attempted immediately at the site of specimen collection in operating theaters and then incubated under aerobic and anaerobic conditions without using a transport medium. All the inoculated culture media were held at a temperature between $35 C^{\circ}-37 C^{\circ}$ for 24 hours, and with further 48 hours incubation if growth negative.

Identification of the isolates was relied on their colonial morphology, gram stain, and standard biochemical tests ${ }^{(11)}$.
Table (1): The age and sex distribution of 184 patients with chronic osteomyelitis.

\begin{tabular}{|l|c|c|c|c|c|c|}
\hline \multirow{2}{*}{$\begin{array}{c}\text { Age } \\
\text { (years) }\end{array}$} & \multicolumn{4}{|c|}{ Gender } & \multicolumn{2}{|c|}{ Total } \\
\cline { 2 - 7 } & \multicolumn{2}{|c|}{ males } & \multicolumn{2}{c|}{ females } & \multicolumn{2}{|c|}{} \\
\cline { 2 - 7 } & No & $\%$ & No & $\%$ & No & $\%$ \\
\hline$<15$ & 28 & $15.2 \%$ & 15 & $8.1 \%$ & 43 & $23.3 \%$ \\
\hline $15-30$ & 32 & $17.3 \%$ & 7 & $3.8 \%$ & 39 & $21.2 \%$ \\
\hline$>30$ & 81 & $44 \%$ & 21 & $11.4 \%$ & 102 & $55.5 \%$ \\
\hline Total & 141 & $76.6 \%$ & 43 & $23.4 \%$ & 184 & $100 \%$ \\
\hline
\end{tabular}

\section{Statistical analysis}

Results are reported as mean \pm standard deviation. The unpaired student $(t)$ test used to calculate the differences between two means. The $p$ value was considered significant if it was less than 0.05 . The reliability calculated by $2 \times 2$ table; the reliability $=A+D /$ Total $\times 100$. Statistical analysis were conducted by using the: Statistical Package for Social Sciences for window, version 11 (SPSS Inc. Chicago Illinois USA).

\section{Results}

Highest incidence of the disease was seen in patients over 30 years (55.5\%). The majority of the current cases of chronic osteomyelitis were involve the long bones specially femur and tibia (64.6\%); as shown in (table 2). Chronic osteomyelitis follow posttraumatic or postoperative infection (exogenous) in 132 patients (71.7\%), and follow acute haematogenous osteomyelitis (haematogenous) in 52 patients (28.3\%), (Table 3). In 152 patients $(83 \%)$, the presentation was chronic discharging sinuses, and in 32 cases (17.4\%), the presentation was acute on chronic osteomyelitis, (table 3).

Aerobic infections were detected in 134 patients ( $72.8 \%$ ), give 222 isolates ( mixed in $78 \%$ and pure in 22\%). The commonest microorganisms detected were Staph. aureus in 65 isolates (29.3\%), followed by Pseudomonas in 41 isolates (18.5\%), E. coli in 35 isolates (15.8\%), Staph. epidermidis in 31 isolates (14\%), Proteus in 22 isolates (9.9\%), and other microorganisms in 27 isolates (12.6\%), (table 4). 
Table (2): The distribution of anatomical location of different types of chronic osteomyelitis involvement.

\begin{tabular}{|l|c|c|c|c|c|c|c|c|}
\hline \multirow{2}{*}{ Bone affected } & \multirow{2}{*}{ Aerobic infection } & \multicolumn{4}{|c|}{ Anaerobic infection } & \multicolumn{2}{|c|}{} \\
\cline { 2 - 8 } & & & \multicolumn{2}{|c|}{ Pure } & \multicolumn{2}{|c|}{ Mixed } & \multicolumn{2}{|c|}{ Total } \\
\cline { 2 - 9 } & No & $\%$ & No & $\%$ & No & $\%$ & No & $\%$ \\
\hline Femur & 48 & $26.1 \%$ & 6 & $3.3 \%$ & 11 & $6 \%$ & 65 & $35.3 \%$ \\
\hline Tibia & 42 & $22.8 \%$ & 4 & $2.2 \%$ & 8 & $4.3 \%$ & 54 & $29.3 \%$ \\
\hline Foot bones & 14 & $7.6 \%$ & 2 & $1.1 \%$ & 3 & $1.6 \%$ & 19 & $10.3 \%$ \\
\hline Humerus & 15 & $6.5 \%$ & 2 & $1.1 \%$ & 1 & $0.54 \%$ & 15 & $8.2 \%$ \\
\hline Radius & 9 & $4.8 \%$ & 0 & & 4 & $2.2 \%$ & 13 & $7.0 \%$ \\
\hline Ulna & 3 & $1.6 \%$ & 0 & & 2 & $1.1 \%$ & 5 & $3.0 \%$ \\
\hline Pelvic bones & 1 & $0.54 \%$ & 1 & $0.54 \%$ & 2 & 1.1 & 4 & $2.0 \%$ \\
\hline Fibula & 3 & $1.6 \%$ & 0 & & 1 & $0.54 \%$ & 4 & $2.0 \%$ \\
\hline Other bones * & 2 & $1.1 \%$ & 1 & $0.54 \%$ & 2 & $1.1 \%$ & 5 & $3.0 \%$ \\
\hline Total & 134 & & 16 & & 34 & & 184 & $100 \%$ \\
\hline
\end{tabular}

${ }^{*}$ Clavicle, patella, metacarpal.

Table (3): Distribution of clinical presentation according to the cause of chronic osteomyelitis.

\begin{tabular}{|l|c|c|c|c|c|c|}
\hline \multirow{2}{*}{$\begin{array}{c}\text { Cause of chronic } \\
\text { osteomyelitis }\end{array}$} & \multicolumn{2}{|c|}{ Acute on chronic } & \multicolumn{2}{c|}{$\begin{array}{c}\text { Chronic discharging } \\
\text { sinus }\end{array}$} & \multicolumn{2}{c|}{ Total } \\
\cline { 2 - 7 } & No. & $(\%)$ & No. & $(\%)$ & No. & $(\%)$ \\
\hline haematogenous & 14 & $7.6 \%$ & 38 & $20.7 \%$ & 52 & $28.3 \%$ \\
\hline exogenous & 18 & $9.8 \%$ & 114 & $61.9 \%$ & 132 & $71.7 \%$ \\
\hline Total & 32 & $17.4 \%$ & 152 & $82.6 \%$ & 184 & $100 \%$ \\
\hline
\end{tabular}

Table (4): Distribution of aerobic isolates from culture of infected bone debridement.

\begin{tabular}{|c|c|c|c|c|c|c|c|c|c|c|}
\hline \multirow{3}{*}{$\begin{array}{l}\text { Type of aerobic } \\
\text { microorganism }\end{array}$} & \multicolumn{4}{|c|}{ Acute on chronic osteomyelitis } & \multicolumn{4}{|c|}{ Chronic discharging sinus } & \multirow{2}{*}{\multicolumn{2}{|c|}{ total isolates }} \\
\hline & \multicolumn{2}{|c|}{$\begin{array}{c}\text { Following } \\
\star \text { *A.H.O }\end{array}$} & \multicolumn{2}{|c|}{ Exogenous } & \multicolumn{2}{|c|}{$\begin{array}{l}\text { Following } \\
{ }^{*} \text { A.H.O } \\
\end{array}$} & \multicolumn{2}{|c|}{ Exogenous } & & \\
\hline & No. & $(\%)$ & No. & $(\%)$ & No. & $(\%)$ & No. & (\%) & No. & $(\%)$ \\
\hline Staph. aureus & 2 & 0.9 & 10 & $4.5 \%$ & 6 & $2.7 \%$ & 47 & $21.1 \%$ & 65 & $29.3 \%$ \\
\hline Pseudomonas & 2 & $0.9 \%$ & 7 & $3.15 \%$ & 6 & $2.7 \%$ & 26 & $11.7 \%$ & 41 & $18.5 \%$ \\
\hline E.coli & 1 & $0.45 \%$ & 7 & $3.15 \%$ & 5 & $2.25 \%$ & 22 & $9.9 \%$ & 35 & $15.8 \%$ \\
\hline $\begin{array}{l}\text { Staph. } \\
\text { epidermidis }\end{array}$ & 2 & $0.9 \%$ & 6 & $2.7 \%$ & 7 & $3.15 \%$ & 16 & $7.2 \%$ & 31 & $14 \%$ \\
\hline Proteus & 1 & $0.45 \%$ & 3 & $1.35 \%$ & 5 & $2.25 \%$ & 13 & $5.85 \%$ & 22 & $9.9 \%$ \\
\hline $\begin{array}{l}\text { Other micro- } \\
\text { organisms }\end{array}$ & 2 & $0.9 \%$ & 4 & $1.8 \%$ & 6 & $2.7 \%$ & 16 & $7.2 \%$ & 28 & $12.6 \%$ \\
\hline total & 10 & $4.5 \%$ & 37 & $16.7 \%$ & 35 & $15.8 \%$ & 140 & $63 \%$ & 222 & $100 \%$ \\
\hline
\end{tabular}

${ }^{*} \mathrm{~A} . \mathrm{H} . \mathrm{O}$ is acute haematogenous osteomyelitis

Anaerobic organisms were detected in 50 cases $(27.2 \%)$ out of the total 184 cases of chronic osteomyelitis, and 58 different anaerobic bacterial isolates were recovered.
Peptostreptococcus; represented the most frequent isolate $(34.9 \%)$, followed by Bacteroides (22.1\%) Prevotella. melaninogenicus (17.2\%) Propionibacterium. 
acne (12.1\%) Fusobacterium (5.2\%) Clostridium. perfringens (3.4\%), Eubacterium (3.4\% ) and Viellonella (1.7\%), (table 5).

Out of the 20 patients with acute on chronic osteomyelitis following acute haematogenous osteomyelitis, only 2 of them were infected with anaerobic bacteria which yielded 3 bacterial isolates. Among the 14 patients with acute on chronic osteomyelitis following exogenous osteomyelitis, anaerobes were present in 4 cases only and yielded 6 anaerobic isolates. Regarding the 38 patients with chronic discharging sinus following acute haematogenous osteomyelitis, anaerobic bacteria were detected in 14 cases and gave 14 anaerobic isolates, while out of the 114 patients with chronic discharging sinus following exogenous osteomyelitis, 30 revealed anaerobic infections and yielded 33 anaerobic isolates (table 3).

The duration of illness in patients with aerobic infection was 178 days \pm 121 , while that in patients with anaerobic was 890 days \pm 625 , as shown in (Table 6). The difference between the two durations was statistically very highly significant $(p<0.0001)$. This means that anaerobic microorganisms were predominating in cases with long duration of disease than those with short duration of illness. From 50 cases with anaerobic infections; anaerobic organisms were found to be mixed with aerobes in 34 cases (68\%), while the other 16 cases (32\%) yielded pure anaerobes, (table 2).
Pure anaerobic growth was noted in older patients than their counterparts with mixed aerobic and anaerobic isolates; their average age was 57.3 years compared to 38.4 years respectively, the age of patients with pure anaerobic infection is highly significant, $p$ value is less than 0.001 , (table 7). The cultures of chronic osteomyelitis from bone was mixed in 112 patients $(60.9 \%)$ and pure in 72 patients (39.1\%), (table 8) .

Result of cultures from pus aspirates in acute on chronic osteomyelitis was compatible with infected bone debridement culture in 30 cases , and not matched in 2 cases. The reliability of cultures from intra- operative aspirate of pus in comparison with cultures from infected bone debridement in acute on chronic osteomyelitis was $93.7 \%, \mathrm{~m}$ (table 9). Results of cultures from discharging sinuses were compatible with infected bone debridement culture in 65 cases, while not so in 87 cases. The reliability of cultures from sinus discharge in comparison with cultures from infected bone debridement in discharging sinus of chronic osteomyelitis was $42.7 \%$ (table 10). Results of cultures from discharging sinus of patients with anaerobic infection were compatible with infected bone debridement cultures in 13 cases, and incompatible in 37 cases. The reliability of cultures from sinus discharge for anaerobic microorganism in comparison with cultures from infected bone was $26 \%$, (table 11).

Table (5): Distribution of anaerobic isolates from culture of infected bone debridement.

\begin{tabular}{|c|c|c|c|c|c|c|c|c|c|c|}
\hline \multirow{3}{*}{$\begin{array}{c}\text { Types of anaerobic } \\
\text { bacteria }\end{array}$} & \multicolumn{4}{|c|}{ Acute on chronic osteomyelitis. } & \multicolumn{4}{|c|}{ Chronic discharging sinus } & \multirow{2}{*}{\multicolumn{2}{|c|}{ total }} \\
\hline & \multicolumn{2}{|c|}{ haematogenous } & \multicolumn{2}{|c|}{ exogenous } & \multicolumn{2}{|c|}{ haematogenous } & \multicolumn{2}{|c|}{ exogenous } & & \\
\hline & No. & $(\%)$ & No. & $(\%)$ & No. & $(\%)$ & No. & $(\%)$ & No. & $(\%)$ \\
\hline Pepto streptococcus & 2 & $3.4 \%$ & 2 & $3.4 \%$ & 5 & $8.6 \%$ & 11 & $19 \%$ & 20 & $34.9 \%$ \\
\hline Bacteroides & 1 & $1.7 \%$ & 1 & $1.7 \%$ & 4 & $6.9 \%$ & 7 & $12.1 \%$ & 13 & $22.1 \%$ \\
\hline P. melaninog & 0 & - & 1 & $1.7 \%$ & 2 & $3.4 \%$ & 7 & $12.1 \%$ & 10 & $17.2 \%$ \\
\hline P. acne & 0 & - & 1 & $1.7 \%$ & 2 & $3.4 \%$ & 4 & $7 \%$ & 7 & $12.1 \%$ \\
\hline Fusobacterium & 0 & - & 1 & $1.7 \%$ & 0 & - & 2 & $3.4 \%$ & 3 & $5.2 \%$ \\
\hline C. perf & 0 & - & 0 & - & 0 & - & 2 & $3.4 \%$ & 2 & $3.4 \%$ \\
\hline Eubacterium & 0 & - & 0 & - & 1 & $1.7 \%$ & 1 & $1.7 \%$ & 2 & $3.4 \%$ \\
\hline Veillonella & 0 & - & 0 & - & 0 & - & 1 & $1.7 \%$ & 1 & $1.7 \%$ \\
\hline Total & 3 & $5.2 \%$ & 6 & $10.2 \%$ & 14 & $24.1 \%$ & 53 & $61.5 \%$ & 58 & $100 \%$ \\
\hline
\end{tabular}


Table (6): Comparison of duration illness in aerobic and anaerobic infection.

\begin{tabular}{|l|c|c|c|c|}
\hline \multicolumn{1}{|c|}{ Type of isolates } & $\begin{array}{c}\text { Mean duration } \\
\text { (days ) }\end{array}$ & $\begin{array}{c}\text { Standard } \\
\text { deviation }\end{array}$ & p- value & significance \\
\hline $\begin{array}{l}\text { Aerobic } \\
\text { microorganisms }\end{array}$ & 178 & 121 & 0.0001 & $\begin{array}{c}\text { Very highly } \\
\text { significant }\end{array}$ \\
\hline $\begin{array}{l}\text { Anaerobic } \\
\text { microorganisms }\end{array}$ & 890 & 625 & \\
\hline
\end{tabular}

Table (7): Comparison between age of patients with pure anaerobic and mixed anaerobic isolates.

\begin{tabular}{|l|c|c|c|c|}
\hline \multicolumn{1}{|c|}{ Type of isolates } & $\begin{array}{c}\text { Mean of patients } \\
\text { age in years }\end{array}$ & $\begin{array}{c}\text { Standard } \\
\text { deviation }\end{array}$ & p-value & Significance \\
\cline { 1 - 3 } Mixed anaerobic & 38.4 & 11.1 & 0.001 & $\begin{array}{c}\text { Highly } \\
\text { Significant }\end{array}$ \\
\hline Pure anaerobic & 57.3 & 8.6 & 0.6 \\
\hline
\end{tabular}

Table (8): Type of cultures according to causes of chronic osteomyelitis.

\begin{tabular}{|l|c|c|c|c|c|c|}
\hline \multirow{2}{*}{ Source of osteomyelitis } & \multicolumn{4}{|c|}{ Type of culture } & \multicolumn{2}{c|}{ Total } \\
\cline { 2 - 7 } & \multicolumn{2}{|c|}{ Pure } & \multicolumn{2}{c|}{ Mixed } & \multicolumn{2}{c|}{} \\
\hline Haematogenous & 18 & $9.8 \%$ & 34 & $18.5 \%$ & 52 & $28.3 \%$ \\
\hline Exogenous & 54 & $29.3 \%$ & 78 & $42.4 \%$ & 132 & $71.1 \%$ \\
\hline Total & 72 & $39.1 \%$ & 112 & $60.9 \%$ & 184 & $100 \%$ \\
\hline
\end{tabular}

Table (9): The $2 \times 2$ table of reliability of bones abscesses pus cultures in comparison with bone cultures.

\begin{tabular}{|c|c|c|c|c|}
\hline & & \multicolumn{2}{|c|}{ Bone cultures } & \multirow{3}{*}{$\begin{array}{c}\text { Total } \\
\text { No. (\%) }\end{array}$} \\
\hline & & Positive & Negative & \\
\hline & & No. (\%) & No. (\%) & \\
\hline \multirow{2}{*}{$\begin{array}{l}\text { Discharging sinus } \\
\text { pus cultures }\end{array}$} & Positive & $28(93.3 \%)$ & $0(0 \%)$ & $28(93.3 \%)$ \\
\hline & Negative & $2(6.7 \%)$ & $0(0 \%)$ & $2(6.7 \%)$ \\
\hline \multicolumn{2}{|l|}{ Total: No. (\%) } & $30(100 \%)$ & $0(0 \%)$ & $30(100 \%)$ \\
\hline
\end{tabular}

Table (10): The $2 \times 2$ table for reliability of discharging sinus pus cultures in comparison with bone cultures.

\begin{tabular}{|l|l|c|c|c|}
\hline \multirow{2}{*}{\multicolumn{2}{|c|}{}} & \multicolumn{2}{|c|}{ Bone cultures } & \multirow{2}{*}{$\begin{array}{c}\text { Total } \\
\text { No. (\%) }\end{array}$} \\
\cline { 3 - 4 } & Positive & Negative & \\
\cline { 2 - 4 } & No. (\%) & No. (\%) & \\
\hline \multirow{2}{*}{$\begin{array}{l}\text { Discharging sinus } \\
\text { pus cultures }\end{array}$} & Positive & $65(42.7 \%)$ & $0(0 \%)$ & $65(42.7 \%)$ \\
\cline { 2 - 4 } & Negative & $87(57.3 \%)$ & $0(0 \%)$ & $87(57.3 \%)$ \\
\hline \multicolumn{2}{|l|}{ Total: No. (\%) } & $152(100 \%)$ & $0(0 \%)$ & $152(100 \%)$ \\
\hline
\end{tabular}

Table (11): The $2 \times 2$ table for reliability of discharging sinus pus cultures of anaerobic infections in comparison with bone cultures.

\begin{tabular}{|l|l|c|c|c|}
\hline \multirow{2}{*}{\multicolumn{2}{|c|}{}} & \multicolumn{2}{|c|}{ Bone cultures } & \multirow{2}{*}{$\begin{array}{c}\text { Total } \\
\text { No. (\%) }\end{array}$} \\
\cline { 3 - 4 } & Positive & Negative & \\
\cline { 3 - 4 } & No. (\%) & No. (\%) & \\
\hline $\begin{array}{l}\text { Discharging sinus } \\
\text { pus cultures }\end{array}$ & Positive & $13(26 \%)$ & $0(0 \%)$ & $13(26 \%)$ \\
\cline { 2 - 4 } & Negative & $37(74 \%)$ & $0(0 \%)$ & $37(74 \%)$ \\
\hline \multicolumn{2}{|l|}{\begin{tabular}{l} 
Total: No .(\%) \\
\hline
\end{tabular}}
\end{tabular}




\section{Discussion}

The literature contain variable reports on causative organisms of chronic osteomyelitis. The gold standard etiologic diagnosis of chronic osteomyelitis is bone specimens cultures ${ }^{(5,12)}$. In the current study the ratio of male to female was 3.3:1, this finding may be due to male frequent exposure to accidents, missile injuries, trauma, and fractures. Highest incidence of chronic osteomyelitis was recorded in patients over 30 years (55.5\%) which could be partly attributed to their high hazard of exposure to missile injuries, trauma and infections, as well as the slower healing of fractures compared to younger age groups. The high rate of chronic osteomyelitis follow exogenous causes (posttraumatic or postoperative) in 132 patients (72\%), which means that there was poor control to infections after open fractures and in operating theaters, and the high prevalence of wars injuries in our community following multiple wars. Our finding is similar to that reported in Turkey ${ }^{(13)}$, Germany $^{(14)}$ and Switzerland ${ }^{(15)}$, while the reverse reported in African community ${ }^{(16)}$.

In the current study, anaerobic organisms were isolated from 50 patients (27.2\%) out of 184 cases with pyogenic osteomyelitis, a result that differs from that reported by Malik who reported anaerobes in $2.6 \%$ of chronic osteomyelitis $^{(17)}$, and from that reported by Naumenko et al too, who found anaerobes in $65 \%$ of chronic osteomyelitis ${ }^{(18)}$. This May be explained by differences in climate, environment, community, development, medical services and techniques of bacterial isolation. In the current study, the high incidence of anaerobes indicates the important role of anaerobes in development and persistent of the purulent inflammatory process in chronic osteomyelitis. In a series from Mayo clinic, 40 of 182 patients have surgery for osteomyelitis was found to be anaerobic bacteria in the cultures ${ }^{(4)}$. Anaerobic infection reported in 19\% of osteomyelitis in drug abuser ${ }^{(19)}$, while was no drug abuse detected in our patients. Anaerobic bacteria are increasingly recognized as a potential pathogen in non haematogenous osteomyelitis (20).
The distribution of aerobic isolates in this study was different from that reported by Malik, who reported that the common isolates belongs to Enterobacteriaceae (32.8\%) followed by Staph. aureus in $29.5 \%$, Pseudomonas (15.5\%), anaerobes (2.6\%) and miscellaneous in (19.3\%), which might explained by different community and environment ${ }^{(17)}$. The distribution of aerobic and anaerobic isolates in the current study is different from that reported by Brook and Zuluaga et al , this might explained by different medical facilities, techniques of bacterial isolation, level of development in community and environment ${ }^{(12,21)}$. Some investigators describe the isolation of a strictly anaerobic strain of Staphylococcus epidermidis in pure culture from the site of an infected prosthesis' ${ }^{(20)}$, in our patients we didn't detect this strain.

The cultures of aspirate from bone abscesses is a reliable way in diagnosis, this made aspiration of abscesses a proper way in diagnosis of causative microorganisms and in detecting drug sensitivity. While cultures from discharging sinuses had low diagnostic reliability and it had lower reliability for anaerobic infection. This finding are comparable with that of Zuluaga et al who confirmed that the appropriate diagnosis of chronic osteomyelitis requiring microbiological cultures of the infected bone and non bone specimens are not valid for appropriate diagnosis in chronic osteomyelitis ${ }^{(12,22)}$.

The duration of illness in patients with aerobic infections was significantly shorter than that in with anaerobic infections, which indicates that as the duration of chronic osteomyelitis prolonged, the anaerobic microorganism become predominating. Some investigators mentioned that as the duration of chronic osteomyelitis lengths, the number of isolated species of anaerobes increases ${ }^{(5,10,23)}$. Pure anaerobic growth was more frequent in older patients than their counterparts with mixed aerobic and anaerobic isolates, this may be due to poor circulation in older age which favored anaerobic infection ${ }^{(5,10,24)}$.

We conclude that, chronic osteomyelitis commonly affects adult males in long bones. The exogenous causes are the commonest. 
Aerobic isolates are the common causes, Staph. aureus and Pseudomonas represent the most frequent etiological agents in aerobic osteomyelitis. Anaerobic bacteria are important other cause, Peptostreptococcus and Bacteroides represent the most frequent etiological agents in anaerobic osteomyelitis. Anaerobic culture should be performed routinely for all patients with osteomyelitis. Mixed infection is common. Anaerobes usually isolated in prolonged persistent infection and in older patients. The cultures of aspirate from bone abscesses is reliable way in diagnosis, while cultures from discharging sinuses had low diagnostic reliability and even lower for anaerobic infection.

\section{Reference}

1. Brinker MR, O'connor DP. Orthopedic infection and microbiology. In: Miller M D. Review of orthopaedics. 2004. $4^{\text {th }}$ ed. Saunders. Philadelphia.: 100- 111.

2. Kumar V, Abbas AK, Fausto N, Mitchell RN. Robbins Basic Pathology. $8^{\text {th }}$ ed. Saunders Elsevier. 2007; 810- 11.

3. Brooks GF, Butel JS, Morse SA. Jawetz . Melnick and Adelberg 's"medical microbiology $21^{\text {st }}$ ed. Appleton and Lange, Stanford, Connecticut. 1998; 695-6.

4. Solomon L, principle of fractures treatment. In: Canale S, Beaty J. Campell's operative Warwick DJ, Nayagam S. Apley's System of Orthopaedic and Fractures. 2001. $8^{\text {th }}$ ed. Arnold, London: 27-49.

5. Wood II G. General orthopaedics. 2008, $11^{\text {th }}$ ed. Mosby. Phladelphia: 3017-84.

6. Senneville E, Morant $H$, Descomps $D$, et al. ."needle puncture and trranscutaneous bone biopsy cultures are inconsistent in patients with diabetes and suspected osteomyelitis of the foot. Clin Infect Dis. 2009 ; 48 (7): 888-93.

7. Lewis PR, Sutter VL, Finegold SM, Bone Infections involving anaerobic bacteria. Medicine. 1978; 57: 279-305.

8. Hall, B. B., R. H. Fitzgerald, J .E. Rosenblatt. Anaerobic osteomyelitis. J. Bone Joint Surg. 1983;65:30-35.
9. Spitzer, R.D., $K$.R. Ratzin. Chronic osteomyelitis. South. Med. J. 1991; 84: 671- 672 .

10. Mousa HA, Bakr SS, Hamdan TA. Anaerobic osteomyelitis. Eastern Mediterranean Health Journal. 1996; 2(3):494-500.

11. Murray PR, Baron EJ, Pfaller MA, et al. Manual of clinical of microbiology $7^{\text {th }}$ ed. American society for microbiology.1999. Washington, D.C.: 187-192.

12. Zuluaga AF, Galvis W, Saldarriaga JG, Aguddo M, Salazar BE ,Vesga O. Etiologic diagnosis of chronic osteomyelitis; a prospective study. Arch Intern Med .2006; 166: 95-100.

13. Malik F. Bacterial etiology of osteomyelitis cases at four hospital of Lahore. J Ayub med coll abbottabad. 2003;15:24-7.

14. Naumenko ZS, Rozova LV, Kliushin NU. Involvement of non-clostridial anaerobic microorganisms in pathogenesis of chronic osteomyelitis. Klin lab Diagn 2005; 3: 18-20.

15. Allison DC, Holtom PD, patzakis MJ, Zalavras CG. Microbiology of bone and joint infections in drug abusers. Clin Orthop Relat Res. 2010; 20: 2107-12.

16. Mousa HA. Bone infection. Eastern Mediterranean Health Journal. 2003; 9: 208-14.

17. Brook I. Microbiology and management of joint and bone infection due to anaerobic bacteria .J Orthop Scins. 2008;13:160-9.

18. Rowlinson MC, Lebourgeois $P$, Ward $K Y$, Finegold SM, Bruckner DA. Isolation of a Strictly Anaerobic Strain of Staphylococcus Epidermidis. Journal of Clinical Microbiology. 2006; 857-860.

19. Raff MJ, Melo JC. Anaerobic osteomyelitis. Medicine (Baltimore). 1978;57:83-130.

20. Templeton WC, Warukiewics A, Melo JC. Anaerobic osteomyelitis of long bones. Rev Infect Dis.1983;5:692 -712. 